
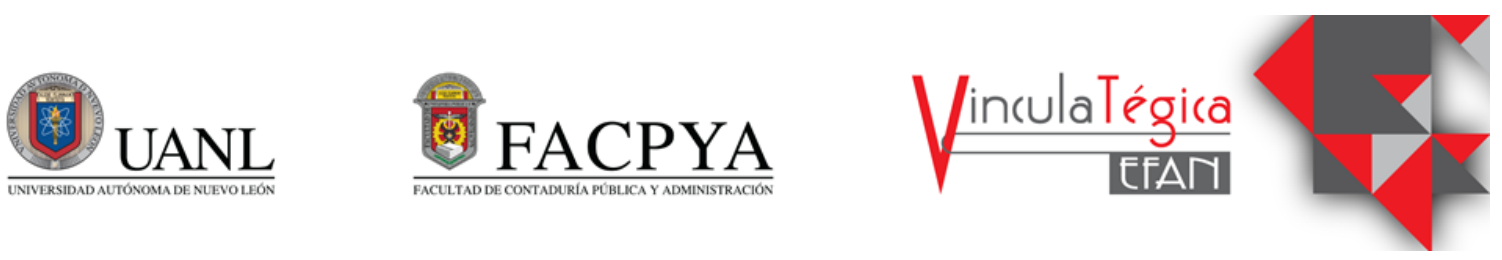

\title{
Uso de simulador virtual como apoyo del aprendizaje del calibre vernier
}

\author{
Daniela del Carmen Bacre Guzmán ${ }^{1}$, Juan Alberto Chi Tapia² y Nury Margarita Leal \\ Rendón ${ }^{3}$
}

'Universidad Autónoma de Nuevo León, Facultad de Ciencias Químicas, Monterrey Nuevo León, México, dbacre@gmail.com, Av. Universidad S/N, Ciudad Universitaria, (+52) 8183294000 ext. 6286

Información del artículo revisado por pares

Fecha de aceptación: junio-2021

Fecha de publicación en línea: diciembre-2021

DOI: https://doi.org/10.29105/vtga7.2-69

\begin{abstract}
Resumen
El uso de simuladores y herramientas virtuales facilita el logro de aprendizajes y la comprensión del uso de equipos cuando no se tienen físicamente, o en cantidades suficientes para cada uno de los alumnos en un salón de clases. Se presentan los resultados obtenidos en la impartición de la unidad de aprendizaje de Normalización y Metrología Dimensional a nivel licenciatura después de haber implementado el uso de un simulador virtual como complemento del aprendizaje del uso de un vernier de forma presencial, y su posterior uso en clases remotas, como apoyo al nuevo programa educativo ajustado a la modalidad a distancia. Se mejoraron los promedios y principalmente la satisfacción y comprensión del uso y lectura del instrumento por parte de los alumnos.
\end{abstract}

Palabras clave: Simulador, Metrología, Educación.

\section{INTRODUCCIÓN}

Es conocido que el uso de simuladores y herramientas virtuales facilita el logro de aprendizajes y la comprensión del uso de equipos cuando no se tienen físicamente, o en cantidades suficientes para cada uno de los alumnos en un salón de clases; aunado a las problemáticas que se presentan en un ambiente de aprendizaje presencial, a los

\begin{abstract}
The use of simulators and virtual tools facilitates the achievement of learning and understanding the use of equipment when it is not physically available, or in sufficient quantities for each of the students in a classroom. The results obtained in the teaching Normalization and Dimensional Metrology subject at undergraduate level are presented after having implemented the use of a virtual simulator as a complement to the learning of the vernier caliper in face-to-face classes, and its subsequent use in remote classes, as support to the new educational program adjusted to the distance modality. The averages and mainly the satisfaction and understanding of the use and reading of the instrument by the students were improved.
\end{abstract}

Key words: Simulator, Metrology, Education.

JEL: I20, I23. estudiantes se les añadió uno más, la imposibilidad de poder tomar las sesiones y laboratorios de forma presencial, y tener que aprender a utilizar los instrumentos de metrología a través de aplicaciones para sesiones de manera remota. Por tanto, en la unidad de aprendizaje (UA) de Metrología Dimensional, se implementó desde hace 
algunos periodos el uso de un simulador virtual como apoyo para el aprendizaje de los estudiantes.

El uso de simuladores en el proceso de educación permite mejorar la calidad de los materiales educativos, así como los programas educativos y métodos pedagógicos innovadores, otorga a los facilitadores oportunidades de construir trayectorias individuales de aprendizaje para los estudiantes. (Vakaliuk, 2020)

El estudio que aquí se presenta busca analizar la contribución de los simuladores virtuales en el aprendizaje de instrumentos de medición, en específico el calibre vernier con estudiantes de ingeniería.

\section{MARCO TEÓRICO}

\subsection{Simulador virtual}

Una opción común que se propone para mejorar los procesos de aprendizaje, de acuerdo con Govea-Valladares et al (2020), es la utilización de simuladores, tomando como ejemplo los maniquíes o cadáveres en la formación de medicina, o los simuladores de vuelo para encarar a los pilotos a situaciones de accidentes o colisiones en escenarios simulados.

En el estudio de Cabero-Almenara (2016) se señala la evolución que se ha tenido de la relación de usuario y las computadoras, ya que en sus inicios fue considerada como un instrumento más, equiparándola con una super calculadora, relación que fue modificada en los ochenta con la llegada de la interfaz Macintosh que permitía la interacción y el dialogo de la computadora con el usuario, además de la navegación y simulación de algunos escenarios. Por otro lado, indica que un simulador forma parte de los distintos tipos de softwares educativos, los cuales deben ser creados con fines didácticos, son usados en una computadora para dar soporte a las actividades de aprendizaje, permiten la interacción además de permitir individualizar el trabajo de cada estudiante, y son los simuladores los que permiten replicar una experiencia o un proceso de la manera más realista y precisa. Además, menciona que los simuladores como apoyo en la educación favorece el aprendizaje por descubrimiento, para lograr que funcione el simulador se debe demostrar lo aprendido y se promueve el aprendizaje individualizado al mismo tiempo que hace más sencilla la autoevaluación.

\subsection{Simuladores de calibre vernier}

Un calibrador vernier es un instrumento que fue diseñado poder hacer uso de un instrumento de lectura directa que permitiera realizar con facilidad una medición, en una sola operación. Cuenta con un vernier o nonio, la cual es una escala auxiliar que se desliza a lo largo de una escala principal lo que permite tener lecturas fraccionales exactas de la mínima división. (González \& Vázquez, 1995)

Al ser el vernier uno de los instrumentos de medición más comunes en el área de la ingeniería, existen distintas opciones de simuladores para este instrumento, que en su mayoría son plataformas 2D que se encuentran en línea y que sólo permiten su uso al tener una conexión de internet; el Centro Nacional de Metrología (CENAM) cuenta son una herramienta virtual de medición, desarrollado por sus investigadores, que permite el movimiento observar la apertura de las mordazas y el deslizamiento del nonio, a la vez que muestra la medición para corroborar la lectura de la misma. (CENAM - División Metrología Dimensional, s. f.)

El sitio de Educaplus tiene un simulador en línea, que mediante la selección de las opciones permite realizar mediciones interiores, exteriores $\mathrm{o}$ de profundidad, permitiendo la apertura de las puntas y el deslizamiento del nonio, aunque sólo permite la simulación de un vernier en milímetros y una sola resolución, además de que las líneas del instrumento no coinciden con la lectura señalada. (Educaplus Calibre Vernier, s. f.).

El portal del profesor Eduardo J. Stefanelli, cuenta con simuladores vernier análogos y de carátula, en milímetros y pulgadas y distintas resoluciones, además que es posible descargar algunos de los simuladores, por lo cual no es necesario tener una conexión a internet para su uso; permite visualizar el movimiento de las puntas como si se tratara del instrumento real, y tiene la opción de mostrar o no la lectura del mismo, así mismo tiene secciones de ejercicios para 
practicar y una de autoevaluación para cada instrumento, y permite además poder acceder desde cualquier dispositivo móvil, lo cual lo hace más atractivo para los estudiantes de licenciatura de hoy en día. (Stefanelli, 2020)

\subsection{La simulación como herramienta para el aprendizaje}

De acuerdo con Márquez (2010), la simulación en sus inicios fue con fines militares ya que eran utilizadas para el entrenamiento profesional de pilotos y presenta a E. Link como el creado del primer simulador de vuelo de tipo comercial en 1930, el cual definía como una mezcla de un dispositivo de entrenamiento para los estudiantes de aviación y de un aparato de entretenimiento, lo que a decir del autor marcaría la doble faceta de los simuladores: el aprendizaje de manera lúdica.

La simulación en la educación ha sido principalmente utilizada en las ciencias médicas con fines educativos y de evaluación, ya que perfecciona el proceso de aprendizaje y permite disminuir las molestias que se generan en los pacientes durante su práctica. El uso de simuladores en los salones de clase apoya la generación de conocimiento de los estudiantes, al hacerlo interactivo y logrando ventajas como la pronta retroalimentación que permite corregir o reforzar las acciones del alumno; de igual manera los simuladores permiten practicar a usuarios que carecen de experiencia en el tema. Se ha encontrado que el uso de simuladores virtuales permite mejorar la retención en el aprendizaje, cuando se utilizan como complemento de los contenidos curriculares, además de que permite flexibilidad de horario a los estudiantes para practicar dichos contenidos. (Contreras \& Carreño, 2012)

De modo que el uso de los simuladores permite trabajar de forma asíncrona a los estudiantes, de acuerdo a Ebner (2020) esto significa que los ejercicios no necesitan ser realizados en tiempo real durante las horas clase, sino que pueden llevarse a cabo a la hora que el alumno les sea más conveniente, pero, aun así, el facilitador debe utilizar tiempo de la clase síncrona para explicar el tema y dar indicaciones de uso del simulador y lo que espera de como práctica por parte de los estudiantes.

Por consiguiente, al hacer uso de un simulador virtual como herramienta de apoyo para el aprendizaje de uso de un vernier, se estaría trabajando en un ambiente virtual de aprendizaje, lo cual Dillenbourg et al. (2002) definen "como una gama de sistemas que comprenden características como un espacio de información diseñado, un espacio social, en donde los participantes son actores activos y presentes, y refieren que un ambiente virtual de aprendizaje pueden ser identificado por medio de las siguientes características: los estudiantes son co-constructores del espacio virtual, no se restringen solo a la educación a distancia; enriquecen las actividades de un salón de clases, la mayoría de los entornos virtuales se superponen con los entornos físicos".

\section{MÉTODO}

El trabajo que aquí se presenta se trata de una investigación descriptiva que tiene como objetivo demostrar los resultados obtenidos de la evaluación del uso y lectura del calibre vernier de alumnos que cursaron la UA de Normalización y Metrología Dimensional en un programa educativo de la licenciatura en Ingeniería Industrial y Administración, después de haber incorporado el uso de un simulador virtual y realizar la adecuación a la modalidad no escolarizada debido a la conocida pandemia por SARS-CoV-2 (COVID 19).

Debido a la cantidad de alumnos que se tienen por grupo, al no contar con un instrumento de práctica para cada uno de ellos, al tiempo destinado para la práctica en el laboratorio, y a que algunos alumnos no llegaban con la suficiente preparación al laboratorio para poder manipular los instrumentos, se inició la búsqueda de estrategias y herramientas que pudieran complementar las actividades de aprendizaje contenidas en el programa de la unidad de aprendizaje, con lo que se encontró que un simulador virtual podría apoyar en lograr mejores resultados en el aprendizaje del uso en específico del calibre vernier.

Se investigaron y utilizaron algunos 
simuladores de manera exploratoria para ver su facilidad de uso, exactitud en las lecturas y variedad de instrumentos que presentaba, sin establecerlo formalmente, pero con la necesidad de la adecuación de los contenidos a educación a distancia, se siguieron los pasos que a continuación se enlistan, considerando ya de manera formal el uso de un simulador virtual en el programa de la unidad de aprendizaje. Cabe mencionar que, para la modificación de contenidos para este elemento de competencia, se utilizó la estrategia educativa del microaprendizaje, la cual propone impartir contenidos de forma fragmentada para aprovechar los lapsos en donde el cerebro humano mantiene su concentración. (Neave et al., 2021).

Fases implementadas:

1) Análisis y revisión de simuladores existentes considerando facilidad de uso, la exactitud de las lecturas, recursos adicionales, permitir la práctica con vernier en distintas resoluciones y unidades. Entre los simuladores analizados, el que cuenta con las mejores características es el que se encuentra en el portal del Prof. Eduardo Stefanelli, que además de tener el simulador del vernier análogo y de carátula, cuenta también con uno para micrómetros, comparador de carátula y goniómetro, además de contener material de mecánica, termodinámica y diseño.

2) Diseño didáctico de actividades de micro aprendizaje para la adecuación de contenidos a la modalidad a distancia. Las actividades se presentan en la tabla 1.

3) Implementación de las modificaciones al programa académico en modalidad no escolarizada.

4) Aplicación de encuesta cualitativa al término del curso a los estudiantes después de haber utilizado el simulador virtual para el vernier, y después haber sido evaluada dicha competencia.

Las actividades que se utilizaron en la adecuación del contenido a modalidad a distancia se presentan en la tabla 1 y a continuación se describen:

Tabla 1. Actividades de micro aprendizaje utilizadas en el curso en modalidad a distancia.

\begin{tabular}{|c|c|c|c|}
\hline 01 & Concepto de Vernier & 07 & $\begin{array}{l}\text { Presentación de simulador y } \\
\text { explicación de uso }\end{array}$ \\
\hline 02 & Concepto de Nonio & 08 & Ejercicios de práctica \\
\hline 03 & $\begin{array}{l}\text { Concepto de resolución del } \\
\text { instrumento }\end{array}$ & 09 & $\begin{array}{l}\text { Autoevaluación con el } \\
\text { simulador }\end{array}$ \\
\hline 04 & Uso vernier 0.02 y $0.05 \mathrm{~mm}$ & & $\begin{array}{l}\text { Construcción de vernier de } \\
\text { cartón }\end{array}$ \\
\hline 05 & $\begin{array}{l}\text { Presentación de simulador y } \\
\text { explicación de uso }\end{array}$ & 11 & Uso del instrumento \\
\hline 06 & Uso vernier 0.001 y $1 / 128 \mathrm{plg}$ & & $\begin{array}{l}\text { Evaluación mediante examen } \\
\text { en línea }\end{array}$ \\
\hline
\end{tabular}

Fuente: Elaboración propia. (Bacre D, Leal N \& Chi J; 2021)

01. Con imágenes se introduce al concepto y reconocimiento de los distintos tipos de vernier

02. Con una presentación se introduce el concepto de nonio, se les muestra el instrumento.
03. Mediante el uso de una video presentación se explica lo que es la resolución o legibilidad del instrumento y se enseña cómo obtenerla, este punto es de gran importancia para poder llevar a cabo la lectura del instrumento. Figura 1.

Figura 1. Video presentación del 
concepto de legibilidad.

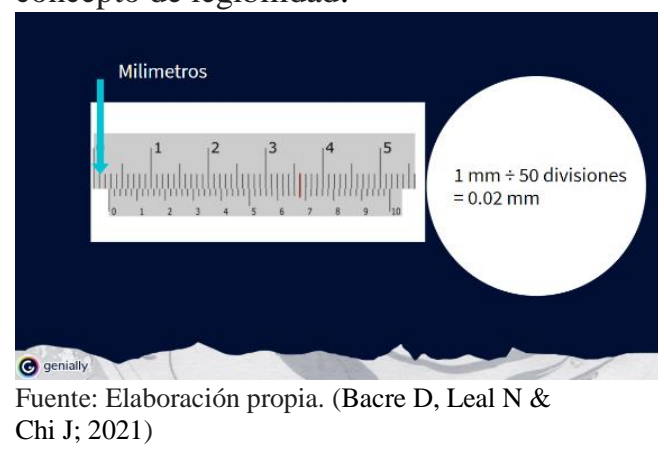

04. Comprendido el punto anterior, se indica como llevar a cabo la lectura del vernier con resolución de 0.02 y 0.05 milímetros.

05. Se muestra al alumno como acceder al simulador en línea, como funciona, la manera de realizar las mediciones para el vernier análogo en milímetros, se invita a utilizarlo para practicar. Figuras 2 y 3.

Figura 2. Página del Prof. Stefanelli.

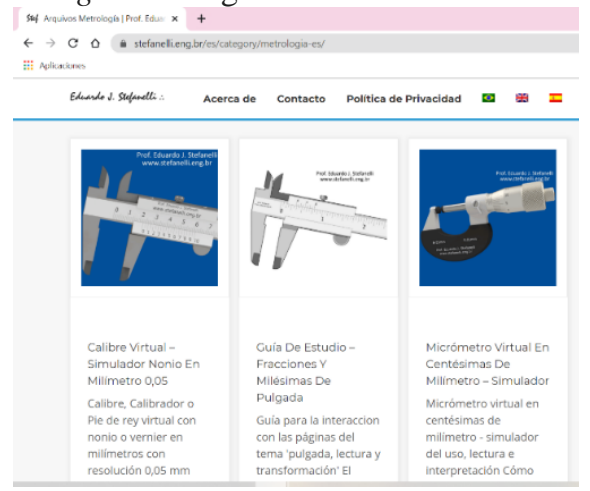

Fuente: Eduardo J. Stefanelli (c) Copyright 2021. Concesión Creative Commons

Figura 3. Simulador vernier de resolución de 0.05 mm. Página del Prof. Stefanelli.

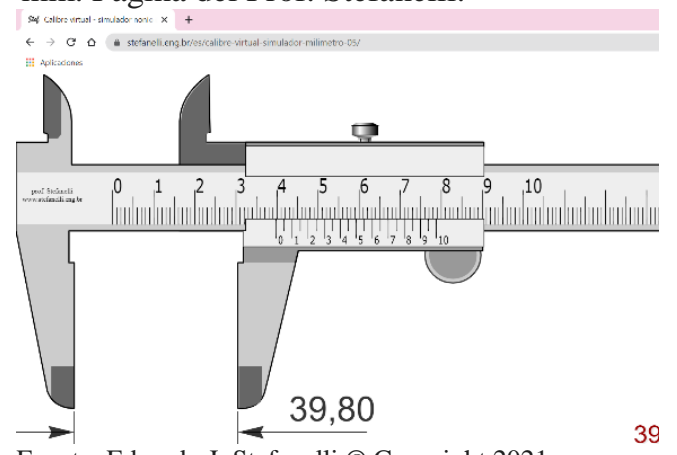

Fuente: Eduardo J. Stefanelli (c) Copyright 2021.

Concesión Creative Commons
06. Se les explica a los alumnos como realizar la lectura del vernier con resolución de 0.001 y $\frac{1}{128}$ de pulgada.

07. Se muestra al alumno como acceder al simulador en línea, como funciona, como realizar las mediciones para el vernier análogo en pulgadas, se invita a utilizarlo para practicar.

08. Se presenta a los alumnos ejercicios de mediciones en milímetros y pulgadas. Se resuelven dudas.

09. Se indica que realicen de manera individual la autoevaluación que se encuentra en la página del Prof. Stefanelli. Figura 4.

Figura 4. Autoevaluación del simulador de vernier.

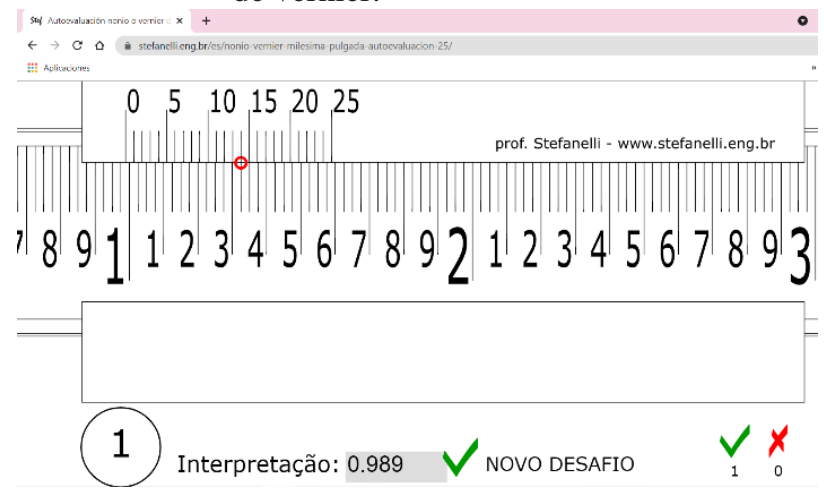

Fuente: Eduardo J. Stefanelli (c) Copyright 2021.

Concesión Creative Commons

10. Como primera evidencia para la evaluación de la competencia, se les proporciona el procedimiento $\mathrm{y}$ plantilla para construir un vernier de cartón, o cualquier otro material que tengan a mano en casa y que les permita hacer mediciones. Figuras 5 y 6.

11. Los estudiantes elaboran un reporte donde presenten como construyeron el instrumento y las mediciones realizadas y de esta forma demostrar que conocen el uso del vernier.

12. Como segunda evidencia para la evaluación de la competencia, responden un examen en línea en donde se les presentan las mediciones y deben indicar las lecturas del calibre vernier. 
Figuras 5 y 6. Elaboración de vernier de cartón y su uso.

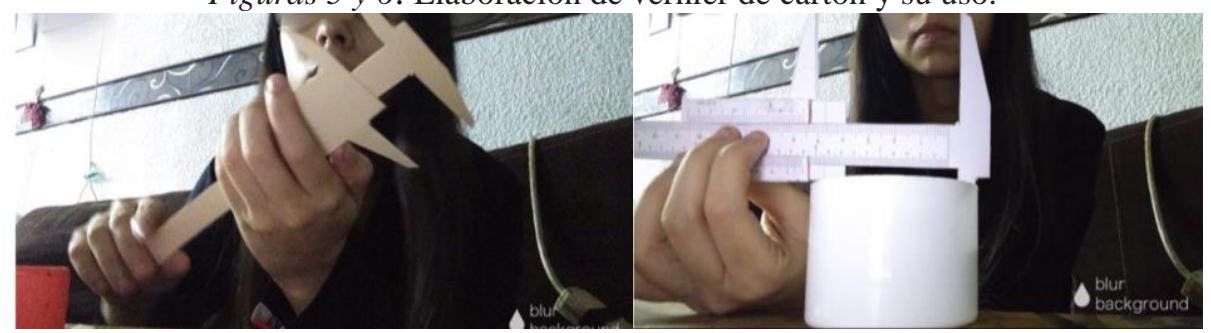

Fuente: Curso de Normalización y Metrología Dimensional. (Bacre D. 2021)

\section{RESULTADOS}

El uso del simulador virtual para el calibrador vernier se empezó a utilizar desde 2019 como un complemento a lo que se veía en el salón de clase, pero se implementó de manera formal a partir de las adecuaciones a los programas derivados del cambio a educación remota, en agosto de 2020, junto a los ajustes a las evidencias para evaluar las competencias de la unidad de aprendizaje de Normalización y Metrología Dimensional, específicamente para el uso del vernier la competencia que se indica en el programa es "Reconocer los calibradores vernier de tipo digital, análogo y digital y realizar mediciones", para lo cual se establecieron dos evidencias con el propósito de evaluar el desarrollo de la competencia. En la figura 7 se puede observar los resultados de las evidencias en los periodos lectivos de agosto - diciembre de 2020 y enero - junio 2021, se obtuvieron mejores resultados durante el primer curso donde se implementaron las estrategias de micro aprendizaje, y con excepción del resultado del examen en el más reciente periodo, los resultados fueron aprobatorios.

Figura 7. Resultados de evidencias utilizadas para evaluar competencia y promedio final.

Evidencias para evaluación de competencia

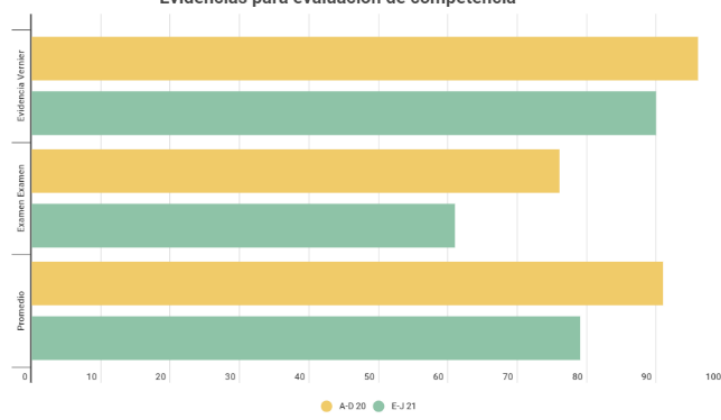

Fuente: Elaboración propia. (Bacre D, Leal N \& Chi J 2021)

En cuanto a la comparativa con los periodos anteriores en donde se utilizaba como complemento el simulador del vernier, la figura 8 presenta los promedios del examen a partir de agosto de 2019 a junio de 2021, se permite ver cómo, a partir de la implementación formal del simulador y las estrategias de micro aprendizaje, se incrementaron los promedios de los alumnos; el injerto muestra el detalle de las calificaciones por alumno del más reciente periodo lectivo.

Figura 8. Promedio de examen para uso de vernier. Injerto: calificaciones por alumno en enero - junio 2021 


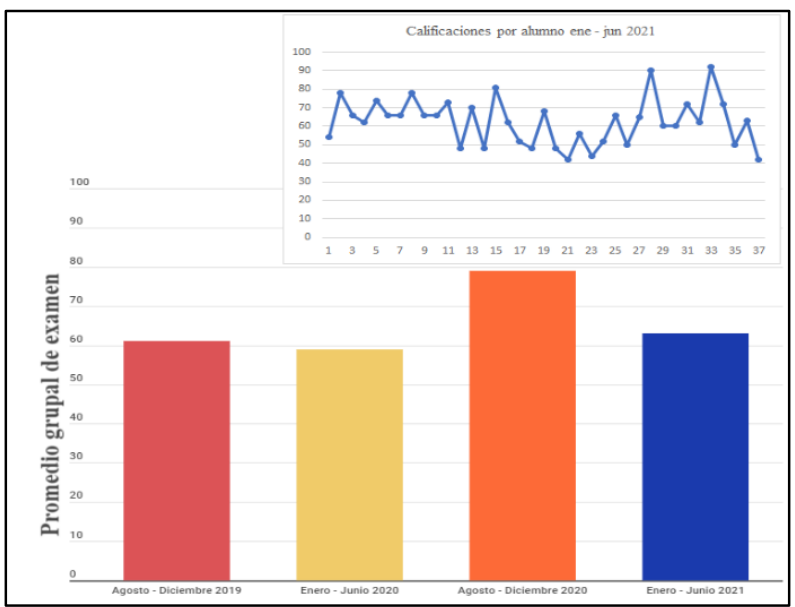

Fuente: Elaboración propia. (Bacre D, Leal N \& Chi J 2021)

Así mismo se desarrolló una encuesta en la que se buscó recabar información al respecto de cómo se sintieron los alumnos al utilizar la aplicación y la forma en la que se llevó el curso en modalidad no escolarizada, la cual se aplicó al término del curso mediante la herramienta en línea de Microsoft Forms.

En la figura 9 muestra que a la pregunta de qué tan probable sería que recomendara el simulador a alguien más, el $67 \%$ de los alumnos indico que sería muy probable que así lo hicieran, mientras que sólo un 3\% dijo que no lo haría; al cuestionarles al respecto de tan seguido utilizaron el simulador el $43 \%$ mencionó que lo utilizaron medianamente, el $31 \%$ muy seguido y un $3 \%$ dijo no haberlo utilizado, además al preguntar si consideraban que el simulador era de ayuda para comprender el uso del calibrado vernier el $85 \%$ respondió que era de mucha ayuda, el $11 \%$ que de mediana ayuda y un $4 \%$ que era de poca ayuda, nadie indicó que no fuera de ayuda.

Figura 9. Respuestas a encuesta aplicada a alumnos al finalizar el curso.

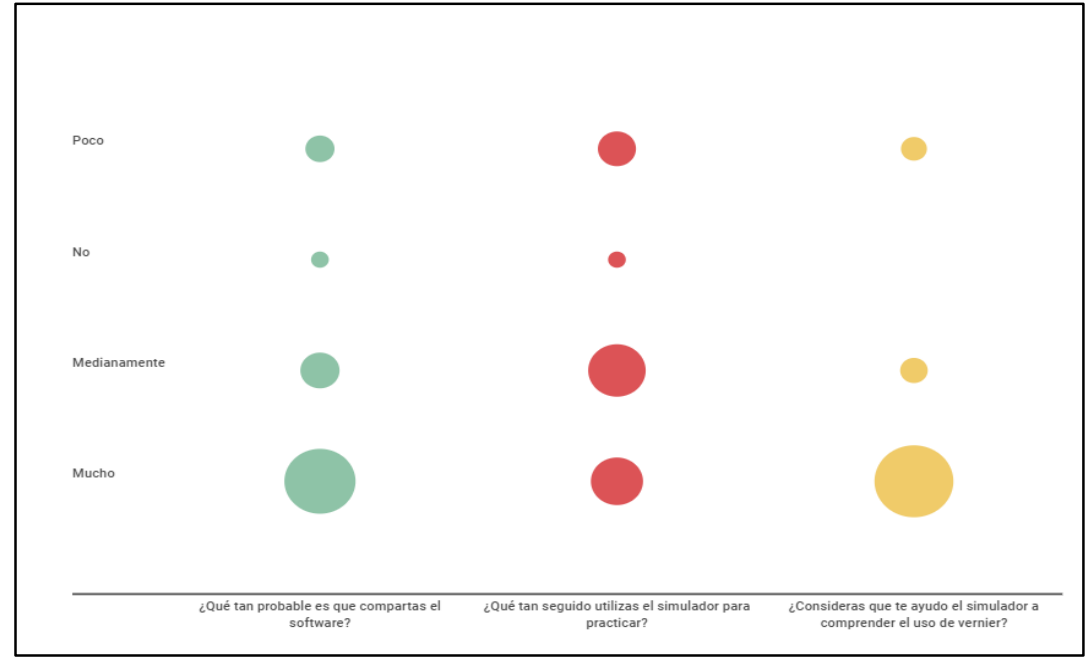

Fuente: Elaboración propia. (Bacre D, Leal N \& Chi J 2021) 
En las figuras 10 y 11 se muestra que el $98 \%$ de los alumnos señalo que haber utilizado le había ayudado a comprender de mejor manera la clase, y al preguntarles si al haber finalizado el curso sabían hacer uso del calibrador vernier el $100 \%$ respondió que sí lo hacían.

Figuras 10 y 11 . Porcentaje de alumnos que indican que el simulador los ayudo a la clase y que saben hacer uso del instrumento.

¿El uso del simulador te ayudo a comprender mejor la clase?

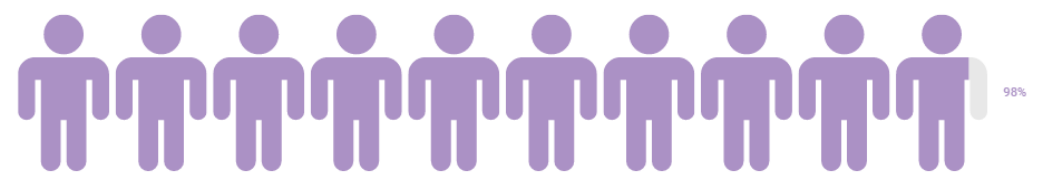

$\mathrm{Al}$ termino del curso, ¿sabes realizar la lectura de un vernier?

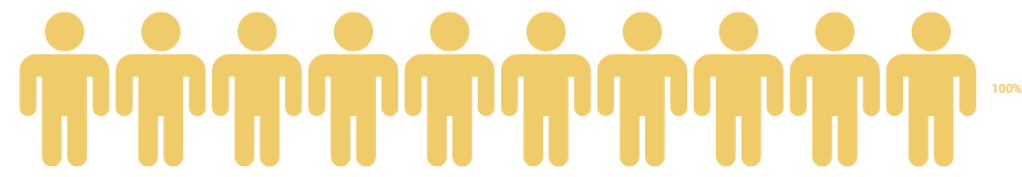

Fuente: Elaboración propia. (Bacre D, Leal N \& Chi J 2021)

Se puede encontrar en la figura 12 que $56 \%$ de los encuestados indico que la experiencia de haber usado el simulador como complemento a sus clases fue muy buena,

Figura 12. Respuestas a cómo fue la experiencia de utilizar el simulador

¿Cómo fue tu experiencia al utilizar el simulador como apoyo en el aprendizaje del uso del vernier? mientras que el $13 \%$ la consideró como poco eficiente.

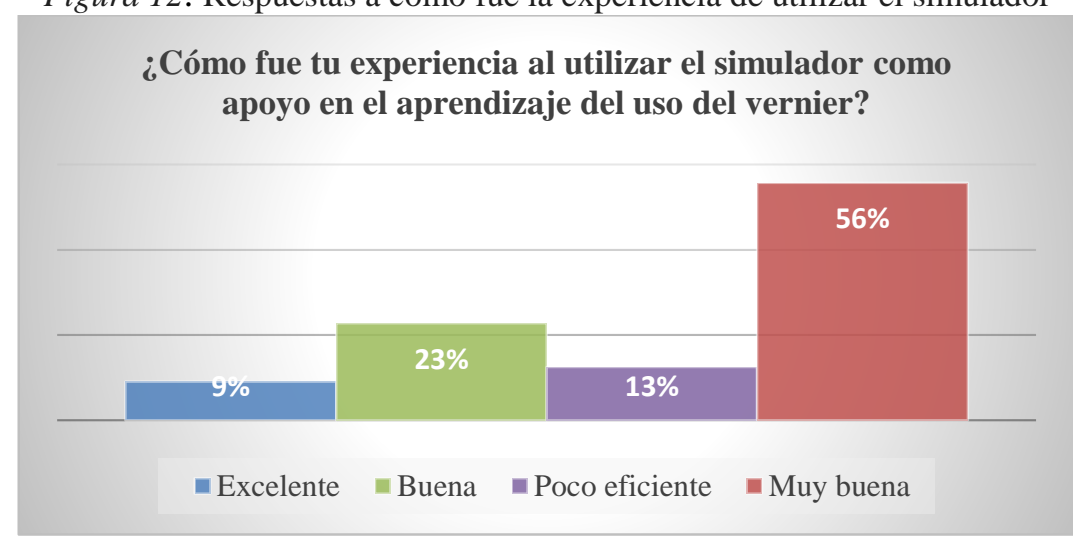

Fuente: Elaboración propia. (Bacre D, Leal N \& Chi J 2021)

De igual manera se les cuestionó a los estudiantes cuales características consideraron mejores al haber usado el simulador, algunas de sus respuestas fueron:

"Que los instrumentos los podemos utilizar cada uno de manera personal"

"El poder usarlos a cualquier hora, sin depender del uso de un laboratorio físico y tener que pedir este con anticipación"

"Que puedes experimentar mucho más y sin riesgo de dañar la herramienta en comparación a la herramienta física"

"Que tengo como comprobar los resultados y aclarar mejor mis dudas"

"Que las herramientas virtuales en el apoyo en el aprendizaje de metrología son muy 
prácticas, que no necesitas comprar el instrumento, ni tenerlo físicamente en tu casa para aprender a utilizarlo, ya que en el simulador puedes aprender"

"Las autoevaluaciones de los instrumentos"

"Que puedo practicar con simuladores si tengo alguna duda en el uso de un instrumento"

"Teniendo las bases teóricas de las clases, he comprendido mejor cuando hay laboratorio y el uso de herramientas, porque de cierta manera me permiten ver aplicado lo aprendido"

"Fue buena idea, además con la realización del vernier casero podía yo manejar el vernier".

$\mathrm{Al}$ preguntarles cual había sido lo más gratificante de haber usado el simulador como apoyo para la impartición de la unidad de aprendizaje, como se muestra en la figura 13, el $74 \%$ menciona que el haberse dado cuenta que sí sabían usar el vernier como lo más gratificante de la experiencia, por otro lado, el $5 \%$ indicaba como lo más gratificante características como la facilidad para ver las medidas en la pantalla y que se podía utilizar el zoom para una mejor lectura del instrumento y el que la página te permitía practicar con las distintas resoluciones del vernier y con otros instrumentos como el micrómetro.

Figura 13. Porcentaje de respuestas a cuál fue lo más gratificante de usar el simulador

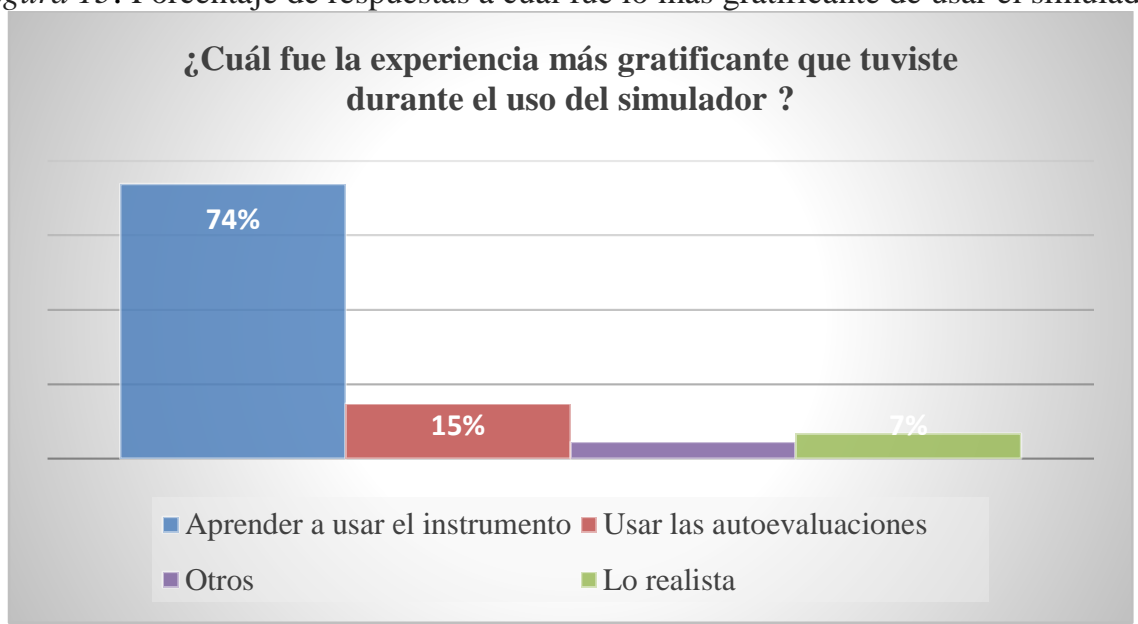

Fuente: Elaboración propia. (Bacre D, Leal N \& Chi J 2021)

Ahora bien, en cuanto pasar una mala experiencia al haber practicado con el simulador del vernier, la figura 14 presenta que el $74 \%$ reporta no haber tenido una mala experiencia, mientras que el $20 \%$ indica que tuvo problemas de conexión o que la página se congelaba, y el resto dijo que la dificultad para aprender a utilizar el simulador había sido lo más negativo de su experiencia.

Figura 14. Porcentaje de respuestas malas experiencias de usar el simulador

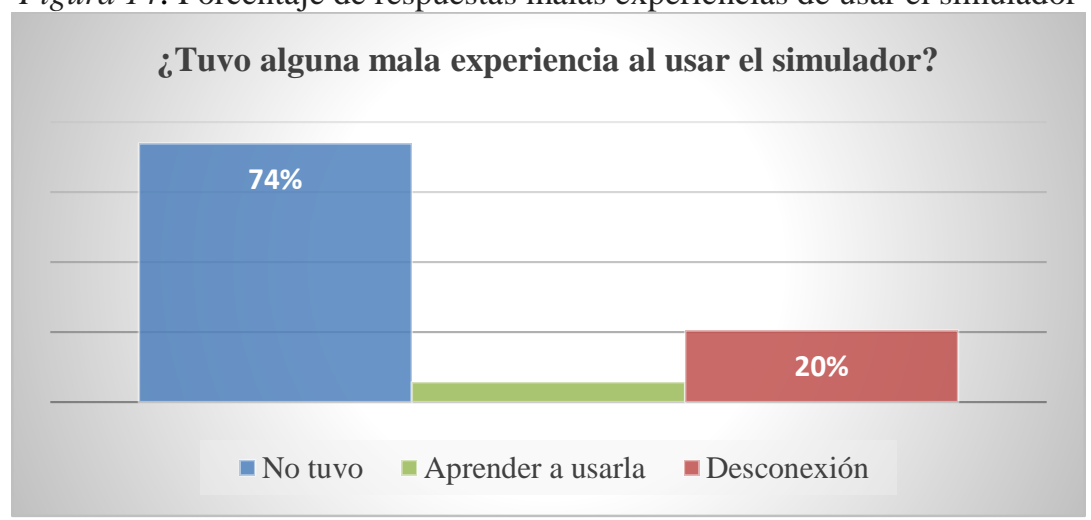

Fuente: Elaboración propia. (Bacre D, Leal N \& Chi J 2021) 


\section{CONCLUSIONES}

El uso de un simulador virtual de un calibre vernier por sí solo no garantiza la mejora del proceso de aprendizaje de los alumnos; sin embargo, como complemento a un correcto diseño de estrategia de aprendizaje resulta ser una herramienta poderosa para facilitar la comprensión del uso y su aplicación, sobre todo en un momento como el presente, en donde aún no es posible tomar las clases de manera presencial, para una unidad de aprendizaje como Normalización y Metrología Dimensional, para la cual es necesario asistir físicamente al laboratorio para poder usar y practicar con los instrumentos señalados en el programa de la misma. Pero más allá del incremento en el promedio de los alumnos, es reconfortante que la mayoría de quienes usaron el simulador señalaron como lo más gratificante significativo del proceso, el comprobar por ellos mismos, y no por un examen formal, que realmente habían aprendido a utilizar e interpretar las lecturas de vernier, ya sea por el uso únicamente del simulador, o por haber utilizado la sección de autoevaluación, a pesar de las bajas expectativas con las que llegaron al curso al saber que no podrían asistir al laboratorio.

Se considera además de importancia la creación de ambientes virtuales de aprendizaje, ya que con las evidencias diseñadas y las actividades de micro aprendizaje implementadas los alumnos logran ser más activos en el logro de su aprendizaje, y puede ser fácilmente trasladado a un salón con clases presenciales, porque el uso de dispositivos móviles en el regreso a la "normalidad" debe de incluirse en los diseños curriculares, para mantener las clases innovadoras, y a los alumnos interesados, de igual manera, la metodología aquí presentada, incluyendo el uso del simulador, podría ser utilizada en el formato de clases hibridas, para que cualquiera que se la opción tomada por el alumno, puedan ser utilizadas las mismas actividades de aprendizaje.

Se debe alentar el uso de simuladores educativos en línea o para descargar en dispositivos móviles porque a los estudiantes los motiva a buscar otras opciones en softwares y aplicaciones para complementar otras unidades de aprendizaje, y les proporciona mayor confianza al asistir al laboratorio, porque ya saben cómo identificar y manipular los instrumentos; pero, por otro lado, esto exige un mayor compromiso por parte de los facilitadores, actualización continua, y una minuciosa preparación de programas educativos que consideren actividades de micro aprendizaje.

Como trabajo a futuro se tiene el implementar una metodología similar para otras competencias a evaluar en la UA de Normalización y Metrología Dimensional. 


\section{REFERENCIAS}

Cabero-Almenara, J. (2016, 17 diciembre). La utilización de simuladores para la formación de los alumnos. Redalyc.org, 17. https://www.redalyc.org/articulo.oa?id=353749552015

CENAM - División Metrología Dimensional. (s. f.). Centro Nacional de Metrología. Recuperado 12 de mayo de 2021, de https://www.cenam.mx/dimensional/simuladores/vernier

Contreras, G. A., \& Carreño, P. (2012, abril). Simuladores en el ámbito educativo: Un recurso $\begin{array}{llll}\text { didáctico para la enseñanza. } & \text { Ingenium, }\end{array}$ https://revistas.usb.edu.co/index.php/Ingenium

Dillenbourg, P., Schneider, D., \& Synteta, P. (2002). Virtual Learning Environments. 3rd Hellenic Conference" Information \& Communication Technologies in Education, 3-18.

Ebner, N. (2020, 19 marzo). Pandemic Pedagogy II: Conducting Simulations and Role Plays in Online, Video-Based, Synchronous Courses by Noam Ebner, Sharon Press: SSRN. Social Science Research Network. https://papers.ssrn.com/sol3/papers.cfm?abstract_id=3557303

Educaplus Calibre Vernier. (s. f.). Educaplus. Recuperado 11 de mayo de 2021, de http://www.educaplus.org/game/calibre

González, C. G., \& Vázquez, J. R. Z. (1995). Metrología. McGraw-Hill Education.

Govea-Valladares, E. H., Martinez-Cruz, M., Yáñez, J. L. H., \& Martinez-Partida, M. (2020). Desarrollo de un Simulador en Realidad Virtual para el uso de una Máquina de Medición por Coordenadas. Academia. https://www.academia.edu/32853659/Desarrollo_de_un_Simulador_en_Realidad_Virtual_p ara_el_uso_de_una_M\%C3\%A1quina_de_Medici\%C3\%B3n_por_Coordenadas

Márquez, I. V. (2010, 4 octubre). La simulación como aprendizaje: educación y mundos virtuales. Libro Nuevos Medios, Nueva Comunicación. https://www.comunicacion3punto0.com

Neave, R., Ibarra, H., \& Báez, L. A. (2021, enero). Micro aprendizaje [Diapositivas]. www.uanl.mx. https://www.uanl.mx

Stefanelli, E. J. (2020b). Metrología. Portal del Prof. Eduardo J. Stefanelli. https://www.stefanelli.eng.br/es/

Vakaliuk, T. (2020, 27 octubre). Using Game Simulator Software Inc in the Software Engineering Education by Tetiana Vakaliuk, Valerii Kontsedailo, Dmytro Antoniuk, Olha Korotun, Iryna Mintii, Andrey Pikilnyak: SSRN. https://papers.ssrn.com/sol3/papers.cfm?abstract_id=3719840 